\title{
SEGURANÇA DO PACIENTE: ANÁLISE DOS GRUPOS DE PESQUISA
}

\author{
Aline Natalia Domingues ${ }^{1}$, Lilian Regina de Carvalho², Sílvia Helena Zem-Mascarenhas ${ }^{3}$
}

RESUMO: O objetivo desse estudo foi verificar as características dos grupos de pesquisa do Conselho Nacional de Desenvolvimento Científico e Tecnológico que trabalham com a segurança do paciente na área da saúde e na enfermagem. Trata-se de um estudo documental, exploratório-descritivo, de natureza quantitativa, realizado por meio do acesso de todos os grupos de pesquisa do Brasil cadastrados no Diretório dos Grupos de Pesquisa do site institucional. A coleta de dados ocorreu em dezembro de 2015, os dados foram armazenados em planilha da Microsoft Excel ${ }^{\circledR}$ e a análise resultou na identificação de 22 grupos de pesquisa. A mobilização e busca do conhecimento articulado com a prática assistencial, ensino e pesquisa são essenciais para se realizar e desenvolver ações que transformem o contexto da saúde em âmbito nacional, tendo a segurança do paciente como principal objetivo.

DESCRITORES: Segurança do paciente; Enfermagem; Grupos de pesquisa; Educação; Pesquisa em Enfermagem.

\section{PATIENT SAFETY: ANALYSIS OF RESEARCH GROUPS}

\begin{abstract}
The present study aimed to assess the characteristics of research groups of theBrazilian National Council for Scientific and Technological Development specialized in patient safetyin health and nursingfields. Documentary, descriptive exploratory study of quantitative approach carried out through access to all Brazilian research groups registered at the CNPq Research Groups Directory.Data collection occurred in December 2015; data were stored in a Microsoft Excel spreadsheet ${ }^{\oplus}$ and analysis resulted in the identification of 22 research groups. Mobilization and search for knowledge combined with care practice, teaching and research are essential to develop actions that improve the healthcare system at the national level, with the primary objective of ensuring patient safety.
\end{abstract}

DESCRIPTORS: Patient Safety; Nursing; Research Groups; Education; Research in Nursing.

\section{SEGURIDAD DEL PACIENTE: ANÁLISIS DE LOS GRUPOS DE INVESTIGACIÓN}

RESUMEN: Estudio cuya finalidad fue verificar las características de los grupos de investigación del Consejo Nacional de Desarrollo Científico y Tecnológico que se dedican a la seguridad del paciente en área de salud y enenfermería. Es un estudio documental, exploratorio y descriptivo, de naturaleza cuantitativa, realizado por medio del acceso de todos los grupos de investigación de Brasil registrados en el Directorio de los Grupos de Investigación del site institucional. Los datos fueron obtenidos en diciembre de 2015 y almazenados en planilla da Microsoft Excel ${ }^{\circledast}$ y el análisis resultó en la identificación de 22 grupos de investigación. La movilización y búsqueda del conocimiento articulado con la práctica asistencial, enseñanza y investigación son esenciales para realizar y desarrollar acciones que cambien el contexto de la salud en ámbito nacional, siendo la seguridad del paciente el principal objetivo. DESCRIPTORES: Seguridad del paciente; Enfermería; Grupos de investigación; Educación; Investigación en Enfermería.

${ }^{1}$ Enfermeira. Mestranda em Ciências da Saúde pelo Programa de Pós-Graduação em Enfermagem, da Universidade Federal de São Carlos. São Carlos, SP, Brasil.

${ }^{2}$ Enfermeira e Mestre. Doutoranda em Ciências da Saúde pelo Programa de Pós-Graduação em Enfermagem da Universidade Federal de Carlos. São Carlos, SP, Brasil.

${ }^{3}$ Enfermeira. Doutora em Enfermagem. Docente de Enfermagem da Universidade Federal de São Carlos. São Carlos, SP, Brasil.

Autor Correspondente:

Aline Natalia Domingues

Universidade Federal de São Carlos

Rd. Washington Luís, km 235, SP-310 - 13565-905 - São Carlos, SP, Brasil

E-mail: alinenataliadomingues@gmail.com
Recebido: 01/03/2016

Finalizado: 10/07/2016 


\section{- INTRODUÇÃO}

O interesse mundial para garantir a segurança na área da saúde voltada à assistência ao cliente está impulsionando o desenvolvimento de políticas, tanto para a melhoria da prática clínica como também o fomento de pesquisas relacionadas à segurança do paciente.

O foco na segurança do paciente, apesar de ser discutido há muitos anos, torna-se evidente somente em 1991 com os resultados do Estudo da Prática Médica de Harvard ${ }^{(1)}$. E em 2000, a segurança no cuidado ao paciente torna-se um dos assuntos prioritários na área da saúde e discutida por pesquisadores de todo o mundo em busca de evidencias científicas ${ }^{(2)}$.

Em 2005 diante da repercussão internacional com a temática, a Organização Mundial da Saúde (OMS) criou o programa denominado Aliança Mundial para a Segurança do Paciente, que tem por objetivo incentivar e divulgar práticas para promover a segurança no cuidado e definir o desenvolvimento de pesquisas baseadas em evidências científicas com melhores práticas voltadas à segurança do paciente ${ }^{(2)}$.

Uma das formas de desenvolver pesquisas é a criação de grupos que são constituídos por pesquisadores, professores, doutores, mestres, especialistas, estudantes e profissionais técnicos interessados em uma mesma área de estudo. Para regular esses grupos, a fim de obter informações em relação às linhas de pesquisa, em 1992 foi criado o diretório de grupos de pesquisa pelo Conselho Nacional de Desenvolvimento Científico e Tecnológico (CNPq), com três principais objetivos: ser instrumento eficiente para intercâmbio e troca de informações; ser fonte inesgotável de informação, e ser uma base de dados que preserve a memória técnico-científica no país ${ }^{(3)}$.

Desta forma, os grupos de pesquisa constituem-se como o lócus de produção de conhecimento, exploram as oportunidades de pesquisa, articulando com as políticas públicas e institucionais ${ }^{(4)}$. Na enfermagem, é um quesito fundamental para que se aplique na assistência estudos desenvolvidos pelos pesquisadores da área, constituindo-se uma importante estratégia para a segurança do paciente ${ }^{(5)}$.

A busca por estudos, estratégias e ações com vistas a garantir a segurança do paciente enquanto está sob os cuidados da equipe de saúde surge mundialmente a partir de 2002 pela Organização Mundial da Saúde $(\mathrm{OMS})^{(6)}$. Ela se tornou responsável por estabelecer normas e dar suporte aos países na elaboração de políticas e práticas relacionadas à segurança do paciente.

Publicações da OMS alertaram a comunidade cientifica sobre os riscos decorrentes da assistência pelos serviços de saúde, assim, diversas áreas da saúde tem buscado desenvolver grupos de estudos e iniciativas voltadas à Segurança do paciente para que a assistência seja prestada com segurança sem causar qualquer dano(7).

No Brasil, o Ministério da Saúde (MS) lança em 2013 o Programa Nacional de Segurança do Paciente ${ }^{(8)}$ (PNSP), que tem por objetivo geral contribuir para a qualificação do cuidado em saúde, instituindo o Comitê de Implementação do Programa Nacional de Segurança do Paciente (CIPNSP), responsável por recomendar estudos e pesquisas relacionados à segurança do paciente.

Diante do exposto, o objetivo deste trabalho foi verificar as características dos grupos de pesquisa do CNPq que trabalham com a segurança do paciente na área da saúde e na enfermagem.

\section{METODOLOGIA}

O estudo corresponde a uma pesquisa do tipo documental, exploratório-descritivo, de natureza quantitativa. A pesquisa documental é realizada a partir de documentos originais (fontes primárias), que ainda não receberam tratamento analítico por nenhum autor, ou seja, não passaram por tratamento científico $^{(9)}$. É um procedimento que se utiliza de métodos e técnicas para a apreensão, compreensão e análise de documentos dos mais variados tipos ${ }^{(10)}$.

As etapas para a coleta documental desta pesquisa descritiva seguiu os seguintes passos: acesso ao site institucional do CNPq (http://www.cnpq.br); em seguida, acesso ao tópico Diretório dos Grupos de Pesquisa onde consta o item Base Corrente; e na tela da Base Corrente foi selecionada a opção Grupos e inserida na caixa de pesquisa a combinação das palavras-chave ${ }^{(11)}$. 
O período da coleta de dados ocorreu no mês de dezembro do ano de 2015, por meio do levantamento dos dados, de todos os grupos de pesquisa do Brasil cadastrados no Diretório dos Grupos de Pesquisa do CNPq versão 5.0 $0^{(3)}$, utilizando-se como palavras-chave: segurança do paciente, saúde, enfermagem e tecnologia na busca paramétrica em sua base corrente. Além disso, como filtro de busca, foi selecionada a opção de grande área sendo ciências da saúde, com o intuito de maximizar e abranger todos os grupos. Para esta busca também foram utilizados e selecionados os filtros da consulta parametrizada, como nome do grupo, nome da linha de pesquisa, palavra-chave da linha de pesquisa e situação de certificado e não atualizado, com a finalidade de aplicar a busca nos campos de consulta dos grupos.

Como critério de inclusão dos grupos de pesquisas, foram considerados os grupos que estavam cadastrados durante este mês de acesso ao site do diretório e certificados pela instituição, relação com o tema de segurança do paciente, seja no nome do grupo de pesquisa ou nas suas respectivas linhas de pesquisa. Incumbe que a certificação dos grupos de pesquisa é de responsabilidade dos dirigentes das atividades de pesquisa da instituição a que o líder do grupo está vinculado(3). Desta forma, adotouse como critério de exclusão os grupos não certificados pela instituição.

A realização destas etapas permitiram a identificação dos grupos de pesquisa que trabalham com a temática da Segurança do Paciente no Brasil. Em uma primeira consulta, foi identificada a existência de 58 grupos de pesquisa sobre o tema. Destes, foram excluídos trinta e seis grupos de pesquisas, por não apresentarem linhas de pesquisa sobre o tema ou não estarem certificados pela instituição.

A Figura 1 ilustra o processo de delineamento da seleção da busca dos artigos. Foram encontrados um total de 22 grupos na base de dados do CNPq com os critérios especificados.

A coleta dos dados foi realizada através do preenchimento de um formulário, as informações foram digitalizadas em uma ferramenta desenvolvida com o aplicativo do Microsoft Office Excel ${ }^{\circledR}$ 2010, com o intuito de elaborar um banco de dados, extraindo os seguintes dados: identificação do grupo, média da composição dos integrantes (pesquisadores, estudantes, técnicos), ano de formação dos grupos, área, parceria com instituição estrangeira, instituição e estado.

Os dados obtidos foram agrupados em forma de tabelas e gráficos para melhor visualização de sua representação. Os resultados de todos os grupos de pesquisas foram analisados através de estatística descritiva mediante cálculos de frequência absoluta e relativa, posteriormente discutidos com literatura pertinente.

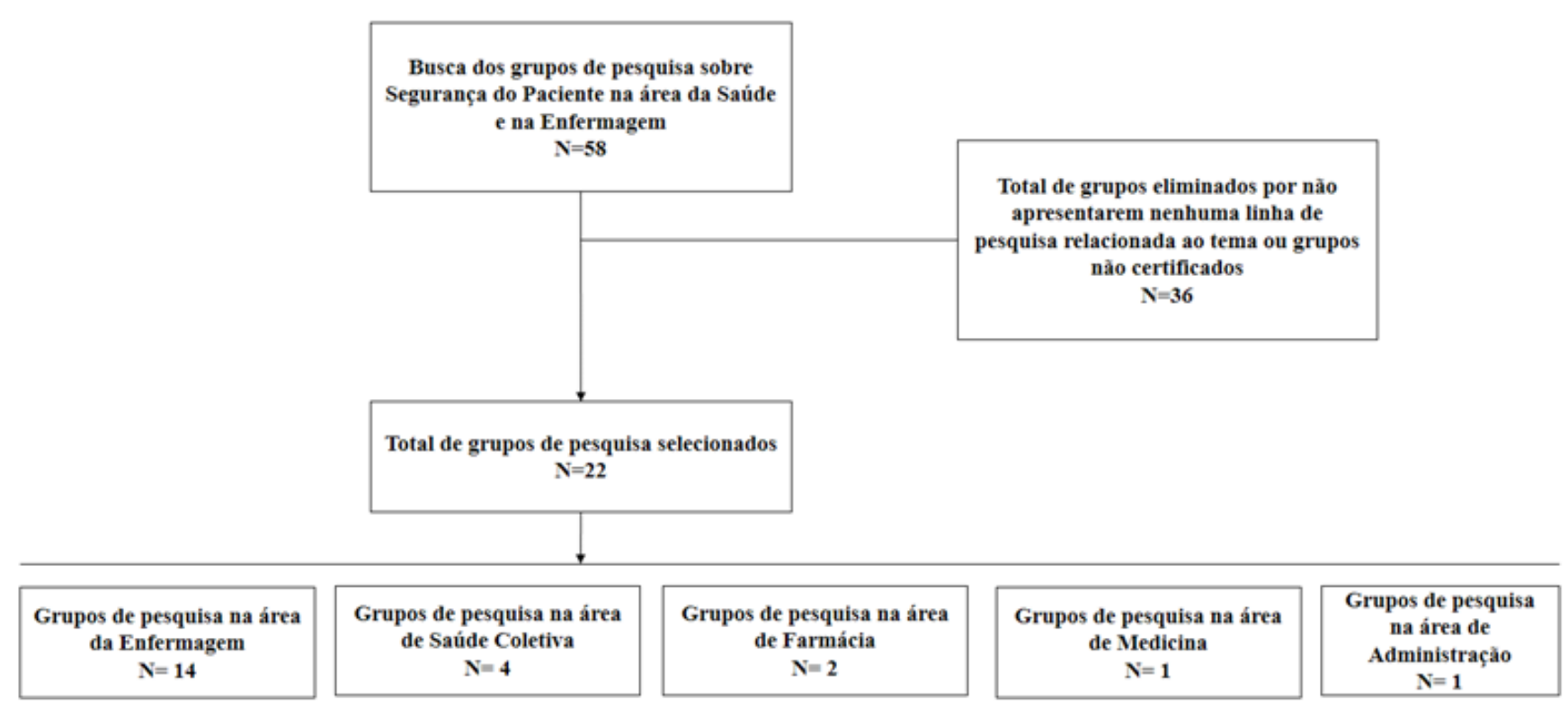

Figura 1 - Processo de delineamento de seleção da busca dos grupos. São Carlos, SP, Brasil, 2016 
Com os dados coletados no Diretório dos Grupos de Pesquisa do site do CNPq, foram selecionados 22 grupos de pesquisa (Tabela 1), resultando um total de 71 linhas de pesquisa sobre o tema.

Tabela 1 - Grupos de pesquisa em Segurança do paciente. São Carlos, SP, Brasil, 2016

\begin{tabular}{|c|c|c|c|}
\hline $\begin{array}{l}\text { Ano de } \\
\text { formação }\end{array}$ & Nome do Grupo & Área & $\begin{array}{l}\text { Instituição de Ensino } \\
\text { Superior (IES)/Sigla }\end{array}$ \\
\hline 1990 & $\begin{array}{l}\text { Grupo Pesquisa Clínica, Tecnologias e Informática em } \\
\text { Saúde e Enfermagem }\end{array}$ & Enfermagem & $\begin{array}{l}\text { Universidade Federal de } \\
\text { Santa Catarina - UFSC }\end{array}$ \\
\hline 1991 & $\begin{array}{l}\text { Núcleo de Estudos e Pesquisa de Enfermagem em } \\
\text { Prevenção e Controle de Infecções Relacionadas à } \\
\text { Assistência à Saúde }\end{array}$ & Enfermagem & $\begin{array}{l}\text { Universidade Federal de } \\
\text { Goiás - UFG }\end{array}$ \\
\hline 1993 & Enfermagem em Unidade de Terapia Intensiva (UTI) & Enfermagem & $\begin{array}{l}\text { Universidade de São Paulo } \\
\text { - USP }\end{array}$ \\
\hline 1996 & $\begin{array}{l}\text { Núcleo de Estudos e Pesquisas em Infecções Relacionadas } \\
\text { ao Cuidar em Saúde - NEPIRCS }\end{array}$ & Enfermagem & $\begin{array}{l}\text { Universidade Federal de } \\
\text { Minas Gerais - UFMG }\end{array}$ \\
\hline 2000 & Assistir/Cuidar em Enfermagem & Enfermagem & $\begin{array}{ll}\text { Universidade } & \text { de } \\
\text { Pernambuco - UFPE } & \\
\end{array}$ \\
\hline 2004 & $\begin{array}{l}\text { Grupo de pesquisas de enfermagem em segurança } \\
\text { do paciente, cuidados intensivos pediátricos e terapia } \\
\text { intravenosa e medicamentosa }\end{array}$ & Enfermagem & $\begin{array}{l}\text { Universidade Federal de } \\
\text { São Paulo - UNIFESP }\end{array}$ \\
\hline 2006 & Saúde do adulto e família & Enfermagem & $\begin{array}{l}\text { Universidade Estadual do } \\
\text { Ceará - UECE }\end{array}$ \\
\hline 2010 & $\begin{array}{l}\text { Laboratório de Estudos e Pesquisas Multidisciplinares em } \\
\text { Segurança do Paciente }\end{array}$ & Enfermagem & $\begin{array}{l}\text { Universidade de Brasília - } \\
\text { UnB }\end{array}$ \\
\hline 2010 & $\begin{array}{l}\text { Laboratório de investigação do cuidado, segurança, } \\
\text { tecnologias em saúde e enfermagem }\end{array}$ & Enfermagem & $\begin{array}{l}\text { Universidade Federal do Rio } \\
\text { Grande do Norte - UFRN }\end{array}$ \\
\hline 2010 & $\begin{array}{l}\text { Saúde Baseada em Evidências na Amazônia: Avaliação de } \\
\text { Tecnologias em Saúde }\end{array}$ & $\begin{array}{l}\text { Saúde } \\
\text { Coletiva }\end{array}$ & $\begin{array}{l}\text { Universidade Federal do } \\
\text { Amazonas - UFAM }\end{array}$ \\
\hline 2010 & $\begin{array}{l}\text { Núcleo de Estudos, Eventos, Pesquisas e Extensão em } \\
\text { Saúde }\end{array}$ & $\begin{array}{l}\text { Saúde } \\
\text { Coletiva }\end{array}$ & $\begin{array}{l}\text { Universidade } \text { Federal do } \\
\text { Acre-UFAC }\end{array}$ \\
\hline 2010 & $\begin{array}{l}\text { Núcleo de Estudos de Enfermagem em Gestão de } \\
\text { Instituições de Saúde e Segurança do Paciente - NEGISP }\end{array}$ & Enfermagem & $\begin{array}{l}\text { Universidade Federal de } \\
\text { Goiás - UFG }\end{array}$ \\
\hline 2012 & Rede de cuidados de Enfermagem aos pacientes críticos & Enfermagem & $\begin{array}{l}\text { Universidade Salgado de } \\
\text { Oliveira - UNIVERSO }\end{array}$ \\
\hline 2013 & $\begin{array}{l}\text { Assistência Farmacêutica: estratégias para melhorar a } \\
\text { segurança do paciente }\end{array}$ & Farmácia & $\begin{array}{l}\text { Universidade } \\
\text { Fluminense - UFF }\end{array}$ \\
\hline 2013 & Gestão em Saúde & Administração & $\begin{array}{l}\text { Universidade Nove de Julho } \\
\text { - UNINOVE }\end{array}$ \\
\hline 2014 & Cuidados Farmacêuticos para Segurança do Paciente & Farmácia & $\begin{array}{l}\text { Universidade } \\
\text { Fluminense - UFF }\end{array}$ \\
\hline 2014 & Gestão e segurança assistencial & Medicina & $\begin{array}{l}\text { Universidade Federal de } \\
\text { Minas Gerais - UFMG }\end{array}$ \\
\hline 2014 & Grupo de Ensino e Pesquisa em Segurança do Paciente & Enfermagem & $\begin{array}{l}\text { Universidade de Caxias do } \\
\text { Sul - UCS }\end{array}$ \\
\hline 2015 & $\begin{array}{l}\text { Núcleo de Estudos de Enfermagem em Administração e } \\
\text { Segurança do Paciente (NEASP) }\end{array}$ & Enfermagem & $\begin{array}{l}\text { Hospital de Clínicas de } \\
\text { Porto Alegre - HCPA }\end{array}$ \\
\hline 2015 & $\begin{array}{l}\text { Núcleo de pesquisa, prática e ensino em gestão em saúde } \\
\text { - NUPPEGES }\end{array}$ & Enfermagem & $\begin{array}{l}\text { Universidade Estadual de } \\
\text { Maringá - UEM }\end{array}$ \\
\hline 2015 & Saúde Coletiva e Gestão de Organizações de Saúde & $\begin{array}{l}\text { Saúde } \\
\text { Coletiva }\end{array}$ & $\begin{array}{ll}\text { Centro Universitário } \\
\text { Hermínio Ometto de } \\
\text { Arararas - UNIARARAS }\end{array}$ \\
\hline 2015 & Melhoria da Qualidade em Serviços de Saúde - QualiSaúde & $\begin{array}{l}\text { Saúde } \\
\text { Coletiva }\end{array}$ & $\begin{array}{l}\text { Universidade Federal do Rio } \\
\text { Grande do Norte - UFRN }\end{array}$ \\
\hline
\end{tabular}


A enfermagem é a área que mais pesquisa sobre segurança do paciente com quatorze $(64 \%)$ no total e a região sudeste é a que mais tem liderados a pesquisa sobre a temática conforme Figura 2.

Em relação às instituições, as federais são as que mais possuem grupos ou linhas de pesquisa totalizando quatorze $(64 \%)$, seguida por particulares quatro (18\%), estadual três (13,5\%) e um $(4,5 \%)$ grupo de pesquisa é realizado dentro de uma instituição assistencial.

Na Tabela 2 é possível analisar o total e média de integrantes e suas titulações dos grupos de pesquisa. Pode-se observar que os doutores têm liderados os grupos de pesquisa em sua maioria, em três grupos de pesquisa foi verificado que os líderes são mestres.

Outra análise que a pesquisa pode identificar é que nenhum grupo de pesquisa brasileiro tem parcerias com grupos de pesquisa estrangeiras.

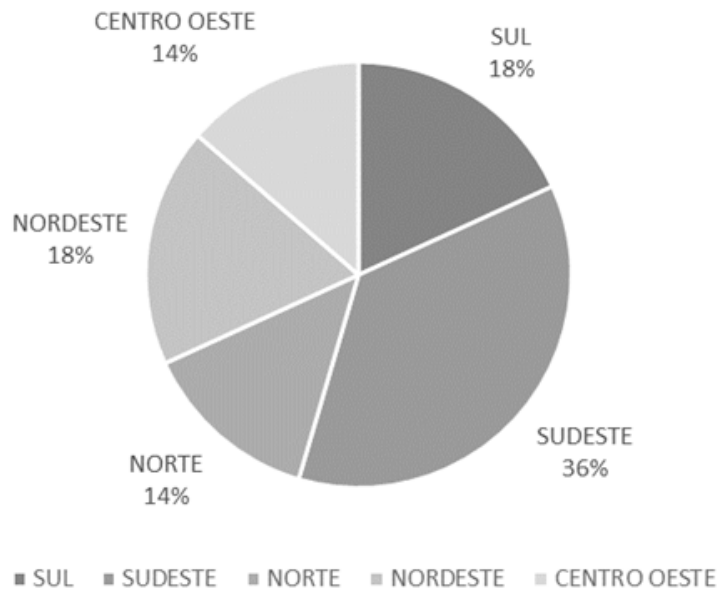

Figura 2 - Distribuição dos grupos por região do Brasil. São Carlos, SP, Brasil, 2016

Fonte: Base de Dados do Diretório dos Grupos de Pesquisa $\mathrm{CNPq}^{(3)}$.

$\mathrm{O}$ ano que mais criou-se grupos ou linha de pesquisa sobre segurança de paciente foi 2010 com cinco (23\%) seguido por 2015 quatro (18\%). A Figura 3 exibe a distribuição do número de grupos criados por ano, relacionados a temática de segurança do paciente.

A área de conhecimento que mais criou grupos ou linhas de pesquisa foi a Enfermagem com quatorze $(63,63 \%)$, seguida por Saúde Coletiva com quatro (18,18\%), Farmácia dois $(9,09 \%)$, Medicina, um (4,54\%) e Administração um (4,54\%).

Tabela 2 - Descrição da característica dos grupos de pesquisa. São Carlos, SP, Brasil, 2016

\begin{tabular}{lcc} 
& Total & Média \\
\hline Linha de Pesquisa & 73 & 3,3 \\
\hline Integrantes & 425 & 19 \\
\hline Doutor & 113 & 5 \\
\hline Doutorando & 28 & 1,3 \\
\hline Mestre & 57 & 2,5 \\
\hline Mestrandos & 43 & 2 \\
\hline Estudantes de Graduação $^{[1]}$ & 43 & 2 \\
\hline Outros $^{[1]}$ & 100 & 2
\end{tabular}

${ }^{[1]}$ Formação acadêmica não informada.

Fonte: Base de Dados do Diretório dos Grupos de Pesquisa $\mathrm{CNPq}^{(3)}$.

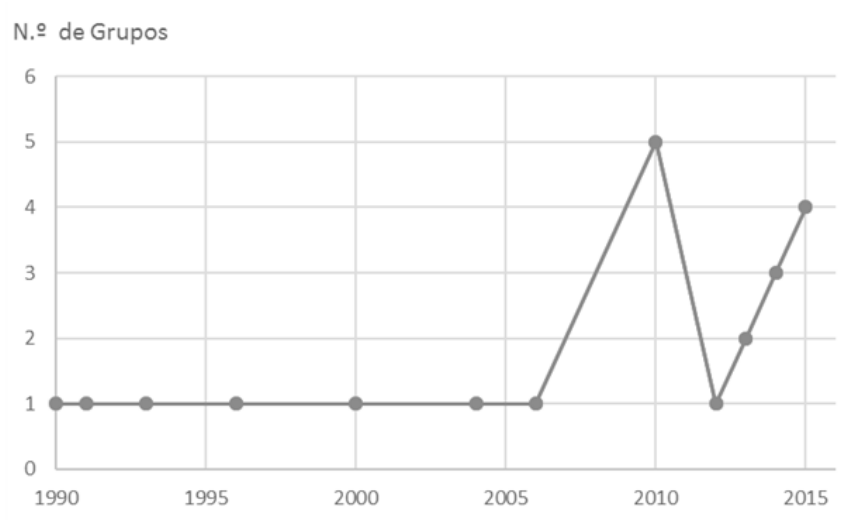

Figura 3 - Distribuição da criação de grupos de pesquisa por ano. São Carlos, SP, Brasil, 2016

Fonte: Base de Dados do Diretório dos Grupos de Pesquisa $\mathrm{CNPq}^{(3)}$.

\section{DISCUSSÃO}

As pesquisas nas instituições de ensino no Brasil estão em expansão e constituem o Diretório dos Grupos de Pesquisa do CNPq, que disponibiliza informações sobre cada grupo em atividade no Brasil. A formação de grupos de pesquisa proporciona a contribuição científica, a troca de informações e experiências entre pesquisadores, o crescimento de uma disciplina científica, além de estímulo à 
publicação ${ }^{(12)}$.

O tema da segurança do paciente tornou-se uma questão relevante para diversos países do mundo a partir do século XXI (13). No Brasil, a preocupação pela segurança do paciente, ainda que incipiente, tem ocorrido desde a década de 90 . Porém, somente nos últimos 5 anos houve maior interesse por estudos nessa temática.

A área de maior concentração de grupos de pesquisa foi representada pela Enfermagem $64 \%$ ( $n=14)$. Esse profissional é responsável por aproximadamente $50 \%$ da mão de obra nos serviços de saúde ${ }^{(14,15)}$. Os profissionais e estudantes vinculados ao ensino vem se mobilizando voluntariamente para a criação de uma rede para discussão da segurança. Assim, em 2008 para criar e desenvolver a REBRAENSP Rede Brasileira de Enfermagem e Segurança do Paciente, estruturada em polos e núcleos cujo objetivo principal é disseminar a cultura de segurança dos pacientes entre pessoas e instituições de saúde ${ }^{(16-17)}$.

Atualmente, quase todos os estados do país têm polos e núcleos da REBRAENSP, criando espaços valiosos de debates com repercussão para as práticas assistenciais, de ensino e de pesquisa ${ }^{(17)}$.

Além disso, há as iniciativas dos órgãos públicos nas instituição de ensino e serviços de saúde que estimulam e incentivam a pesquisa. Dentre eles pode-se citar a Agência Nacional de Vigilância Sanitária (ANVISA), que em $2007^{(18)}$ elaborou uma proposta para identificar os problemas de segurança nos serviços de saúde para se adequar às recomendações da OMS. Esta lançou em 2009(19) o "WHO patient safety research: better knowledge for safer care", indicando os tipos de pesquisa com maior potencial de contribuição para a segurança do paciente, dos quais pode-se citar: (1) mensuração do dano e tipos de danos, (2) compreensão das causas dos incidentes de segurança, (3) desenvolvimento de soluções para a segurança, (4) aprendizagens com a implementação de soluções para a segurança, (5) avaliação do impacto de soluções e (6) transposição de resultados de pesquisas para normas e para a prática ${ }^{(17,19)}$.

Houve também em 2013 o Programa Nacional de Segurança do Paciente do MS e ANVISA, que tem por objetivo a implantação da gestão de risco e de Núcleos de Segurança do Paciente nos estabelecimentos de saúde do país ${ }^{(8)}$.

Outro fator a ser considerado quando se trata da segurança do paciente e que torna a temática de grande relevância para a pesquisa, é o aumento da expectativa de vida da população, diante do significativo avanço tecnológico em relação à prevenção, diagnóstico e tratamento de doenças na área da saúde. Com o envelhecimento da população e a ampliação ao acesso em saúde, a demanda pelos serviços de saúde torna-se imprescindível. Apesar dos esforços das instituições de ensino para a formação dos profissionais da saúde e a prestação da assistência utilizando toda tecnologia disponível, o paciente pode ser colocado em situações de risco e danos ${ }^{(20)}$.

Dados emitidos pelo Censo de 2014 do CNPq demonstram que 43,9\% dos grupos de pesquisa estão localizados na região sudeste. Vale ressaltar que mais da metade dos enfermeiros (53,9\%) do Brasil se concentram nessa região ${ }^{(11)}$.

O CNPq realiza censos bianuais que possibilitam analisar inúmeros dados relacionados aos grupos de pesquisa no Brasil. Segundo os dados de 2014, a distribuição dos pesquisadores segundo a titulação máxima os grupos de pesquisa são formados em sua maioria por doutorados e mestrados, conforme dados encontrados nesse estudo demonstrado na Tabela 2. Além disso, outro estudo também encontrou dados semelhantes a esses ${ }^{(21-22)}$.

Com relação às instituições nas quais os grupos estão inseridos, pode-se observar que a região sudeste do país lidera os grupos de pesquisa corroborando outros achados ${ }^{(9,11,22-25)}$. Essas disparidades entre as regiões no que diz respeito à infraestrutura, corpo docente qualificado e recursos para o desenvolvimento de pesquisas é histórico ${ }^{(12)}$. Os mesmos foram inicialmente implantados em universidades das Regiões Sul e Sudeste, concentrando assim o apoio às pesquisas e garantindo, consequentemente, a produção científica na área ${ }^{(13)}$.

Além disso, autores ${ }^{(23)}$ acreditam que devido à região sudeste representar mais da metade da participação do PIB (Produto Interno Bruto) do país, induz a maior concentração de financiamentos, centros de excelência em pesquisa e recursos humanos. 
Nas últimas décadas, a preocupação com a segurança no cuidado prestado ao paciente tem se tornado um dos assuntos prioritários na área da saúde, refletindo na busca e desenvolvimento de evidências científicas ${ }^{(2)}$, e evidenciando a características dos grupos selecionados de pesquisa que permeiam a área da enfermagem, saúde coletiva, farmácia e medicina.

Como limitação do estudo, aponta-se os grupos de pesquisas analisados, pois os dados coletados constituíram-se via base de dados e não diretamente com os pesquisadores, deixando dúvidas em relação às reais linhas de pesquisas apresentadas, além da necessidade de avaliação do número de publicações apresentadas por cada grupo, relacionado ao tema.

\section{CONCLUSÃO}

O estudo apresentado contemplou a análise dos grupos de pesquisas existentes na área de segurança do paciente, contribuindo para o desenvolvimento da área, possibilitando criação de contatos e fortalecimento da área, além de ressaltar a preocupação da enfermagem na reflexão do tema para sua prática profissional na assistência, no ensino e na pesquisa.

A base de dados do CNPq é fundamental para que a rede de conhecimento seja construída e visualizada no contexto científico. Ademais, os grupos de pesquisa que contam com a participação de diferentes pesquisadores, estudantes e técnicos, podem ampliar as possibilidades de discussões de todos os profissionais da área da saúde, com o intuito de promover uma cultura de segurança do paciente em todos os âmbitos.

As investigações sobre segurança do paciente devem ter como principal meta o desenvolvimento de pesquisas que resultem em diversas estratégias eficazes pelos profissionais da área da saúde, mobilização e busca do conhecimento articulado com a prática assistencial, ensino e pesquisa.

\section{REFERÊNCIAS}

1. Brennan TA, Leape LL, Laird NM, Hebert L, Localio AR, Lawthers AG, et al. Incidence of adverse events and negligence in hospitalized patients. Results of the Harvard Medical Practice Study I. Qual Saf Health Care. [Internet] 1991; 324(6) [acesso em 02 de jan 2016]. Disponível: https://dx.doi.org/10.1136/qshc.2002.003822

2. Cassiani SHB. Enfermagem e a pesquisa sobre segurança dos pacientes. Acta paul. enferm. [Internet] 2010; 23(6) [acesso 02 de jan de 2016];. Disponível: http://dx.doi.org/10.1590/S0103-21002010000600001

3. Conselho Nacional de Desenvolvimento Científico e Tecnológico (CNPq). Base de Dados do Diretório dos Grupos de Pesquisa CNPq. 2015. [acesso em 05 de dezembro de 2015]. Disponível:

http://lattes.cnpq.br/web/dgp/home

4. Erdmann AL, Lanzoni GMM. Características dos grupos de pesquisa da enfermagem brasileira certificados pelo CNPq de 2005 a 2007. Esc. Anna Nery [Internet] 2008; 12(2) [acesso 12 de mar 2016]. Disponível:

http://dx.doi.org/10.1590/S1414-81452008000200018.

5. Fermo VC, Radünz V, da Rosa LM, Marinho MM. Cultura de segurança do paciente em unidade de Transplante de Medula Óssea. Rev. Bras. Enferm. [Internet] 2015; 68(6). Disponível:

http://dx.doi.org/10.1590/0034-7167.2015680620i

6. World Health Organization (OMS). Summary of the evidence on patient safety: implications for research. Geneva; 2008 [acesso em $06 \mathrm{dez}$ 2015]. Disponível: http://www.who.int/patientsafety/information_centre/20080523_ Summary_of_the_evidence_on_patient_safety.pdf

7. Ministério da Saúde (BR), Agência Nacional de Vigilância Sanitária (ANVISA). Segurança do paciente e qualidade em serviços de saúde. Brasília (DF): Ministério da Saúde; 2011.

8. Ministério da Saúde (BR). Portaria n ${ }^{\circ}$. 529, de 1 de abril de 2013. Institui o Programa Nacional de Segurança do Paciente (PNSP). Diário Oficial da União, [Internet] 1 abr 2013 [acesso em 05 jan 2016]. Disponível: http://bvsms. saude.gov.br/bvs/saudelegis/gm/2013/prt0529_01_04_2013.htm 
9. Sá-Silva JR, de Almeida CD, Guindani JF. Pesquisa documental: pistas teóricas emetodológicas. Rev Bras Hist Ciênc Soc. 2009; 1(1): 1-14.

10. Alvez-Mazzotti AJ, Gewandsznajder F. O método nas ciências naturais e sociais. Pesquisa quantitativa e qualitativa. São Paulo: Pioneira Thomson; 1999.

11. Godinho MR, Greco Rm, Gonçalves ÂMC. Enfermagem e Saúde do Trabalhador: análise dos grupos de pesquisa. Rev Rene. 2011; 12(4): 825-32.

12. Perucchi V, Garcia, JCR. Indicadores de produção dos grupos de pesquisa do Instituto Federal de Educação, Ciência e Tecnologia da Paraíba. Revista Brasileira de Biblioteconomia e Documentação [Internet] 2012; 8(1) [acesso 02 de fev 2016]. Disponível: https://rbbd.febab.org.br/rbbd/article/view/193/221

13. Reis CT, Martins M, Laguardia J. A segurança do paciente como dimensão da qualidade do cuidado de saúde: um olhar sobre a literatura. Ciênc. saúde coletiva. [Internet]. 2013; 18(7) [acesso em 05 jan 2016]. Disponível: http://dx.doi.org/10.1590/S1413-81232013000700018

14. Fundação Oswaldo Cruz (FIOCRUZ). Pesquisa inédita traça perfil da enfermagem no Brasil. 2015. [acesso 02 de fev 2016]. Disponível: http://portal.fiocruz.br/pt-br/content/pesquisa-inedita-traca-perfil-da-enfermagem-nobrasil

15. Conselho Federal de Enfermagem (COFEN). Cofen e Fiocruz apresentam Perfil da Enfermagem às Sec. Municipais de Saúde. 2015. [acesso 02 de fev 2016]. Disponível: http://www.cofen.gov.br/cofen-e-fiocruzaprsentam-perfil-da-enfermagem-as-secretarias-municipais-de-saude_33420.html

16. Caldana G, Brito GE, Urbanetto JS, Peterlini MAS, Gabriel CS. Rede brasileira de enfermagem e segurança do paciente: desafios e perspectivas. Texto contexto - enferm. [Internet] 2015; 24(3) [acesso 02 de fev 2016]. Disponível: http://dx.doi.org/10.1590/0104-070720150001980014

17. Urbanetto JS, Gerhardt LM. Segurança do paciente na tríade assistência ensino pesquisa. Rev Gaúcha Enferm. [Internet] 2013; 34(3) [acesso em 01 de abr 2016]. Disponível: http://www.scielo.br/pdf/rgenf/v34n3/a01v34n3.pdf

18. Agência Nacional de Vigilância Sanitária (ANVISA). ANVISA promove debate sobre segurança do paciente no Brasil. Ministério da Saúde. 2007 [acesso 02 de mar 2016]. Disponível:

http://www.anvisa.gov.br/DIVULGA/noticias/2007/171007.htm

19. World Health Organization (WHO). WHO patient safety research: better knowledge for safer care. 2009 [acesso em 15 fev 2016]. Disponível:

http://apps.who.int/iris/bitstream/10665/70145/1/WHO_IER_PSP_2009.10_eng.pdf

20. Pedreira LC, Brandão AS, Reis AM. Evento adverso no idoso em Unidade de Terapia Intensiva. Rev. Brás. Enferm. [Internet] 2013; 66(3) [acesso em 02 de mar 2016]. Disponível: http://dx.doi.org/10.1590/S0034-71672013000300019

21. Pepe VLE, de Noronha ABM, Figueiredo TA, de Souza AAL, Oliveira CVS, Pontes Júnior DM. A produção científica e grupos de pesquisa sobre vigilância sanitária no CNPq. Ciênc. saúde coletiva [Internet] 2010; 15(Supl 3) [acesso em 15 de dez 2015]. Disponível: http://dx.doi.org/10.1590/S1413-81232010000900009

22. Conselho Nacional de Desenvolvimento Científico e Tecnológico (CNPq). Grupos de Pesquisa. 2015. [acesso em 02 de fev 2016]. Disponível: http://lattes.cnpq.br/web/dgp

23. Nickel L, Oliari LP, Vesco SNPD, Padilha MI. Grupos de pesquisa em cuidados paliativos: a realidade brasileira de 1994 a 2014. Esc. Anna Nery [Internet] 2016; 20(1) [acesso em 15 de dez 2015]. Disponível:

http://dx.doi.org/10.5935/1414-8145.20160010

24. Ramos RR, Machado CJS. Uma análise espaço-temporal dos grupos de pesquisa do CNPq: A dengue no Brasil. Hygeia [Internet] 2014; 10(18) [acesso 02 de fev 2016]. Disponível:

http://www.seer.ufu.br/index.php/hygeia/article/view/23174/14819

25. Canever BP, do Prado ML, Backes VMS, Lino MM. Caracterização dos grupos de pesquisa em educação em enfermagem do estado de são paulo. Texto contexto - enferm. [Internet] 2014; 23(1) [acesso em 02 de fev 2016]. Disponível: http://dx.doi.org/10.1590/S0104-07072014000100003 